\title{
The Waveguide Below-Cutoff Attenuation Standard
}

\author{
David H. Russell
}

\begin{abstract}
A semitutorial review of the history, development, and application of waveguide below-cutoff (WBCO) attenuation standards is presented. A brief summary of the electromagnetic (EM) theory is followed by descriptions of the various designs implemented over a span of 60 years. Designs range from simplistic to elaborate electromechanical creations that stretch the toolmaker's art yet are ultimately dependent upon the primary standard of length.
\end{abstract}

Index Terms - Attenuators, calibration, high frequency, measurements, standards, uncertainty, waveguide standards.

\section{INTRODUCTION}

$\mathbf{I}$ N THE CURRENT measurement world of automated vector network analyzers based on multiport reflectometers, it is easy to lose sight of some of the underlying basic standards of power, impedance, and attenuation. The waveguide below-cutoff (WBCO) attenuator propagating a single evanescent mode down a uniform waveguide is nearly an ideal primary standard since incremental attenuation can be closely predicted from only a knowledge of its dimensions within minor restraints of frequency of operation and deviation from losslessness of the material used for fabrication of the guide. Using the device at an intermediate frequency (IF) such as $30 \mathrm{MHz}$ with linear mixing to higher microwave and millimeter wave frequencies, attenuation transfer standards may be calibrated to nearly any desired degree of precision and accuracy. Rotary vane waveguide attenuators are often used for direct attenuation measurements at frequencies above 10 $\mathrm{GHz}$ because their attenuation law can also be theoretically determined as a direct cosine squared function of vane angle. Unfortunately, their inherent mechanical limitations have tended to require cross checking with an IF system using the WBCO attenuator as the absolute reference.

Techniques are required to ensure the validity, in practice, of the theoretical computations used in designing a standard WBCO attenuator. Since attenuation is basically the determination of a dimensionless ratio, methods have been devised using multibranch networks to independently assess the minor errors in WBCO devices due to deviation from losslessness of the guide material and unsuppressed multimode propagation. Other independent methods such as direct power ratio and dc substitution are often used. At the National Institute of Standards and Technology (NIST), exact attenuation steps based

Manuscript received March 31, 1997; revised August 15, 1997.

The author, retired, was with the National Institute of Standards and Technology (NIST), Boulder, CO 80302 USA. He is now at 636 Fairfield Lane, Louisville, CO 80027 USA.

Publisher Item Identifier S 0018-9480(97)08347-6. on a superconducting quantum interference device (SQUID) were successfully used to validate the estimated uncertainty of a WBCO attenuation standard.

\section{HISTORY}

The earliest realizations of this WBCO technology date to the 1930's when Harnett and Case described the use of two moving inductors in a circular tube to test receiver sensitivity [1]. Applications were refined during World War II at the Massachusetts Institute of Technology (MIT) Radiation Laboratory in the United States, where cutoff attenuators with both circular and rectangular cross sections were fabricated with operating frequencies to $24 \mathrm{GHz}$ [2]. This work was rapidly advanced from 1945 to 1950 by Gainsborough, Grantham, Freeman, Wheeler, Brown, Barlow, and Cullen, who refined the earlier crude mechanical designs of WWII and applied appropriate corrections so they could be used as true standards of attenuation [3], [8].

The 1950's through the 1970's saw major thrusts in WBCO design innovation and its establishment as a true primary standard of attenuation through the efforts of such pioneers as Allred, Hollway-Kelly, and Weinschel [9], [14]. These efforts pushed the physical limits of materials and the limits of length metrology to a point of diminishing returns. These "ultimate" WBCO attenuators were developed at NIST [National Bureau of Standards (NBS)] in the United States, the National Physical Laboratory (NPL) in Great Britain, National Measurement Laboratory (NML) in Australia, and in the commercial sector at Weinschel Engineering in the United States. In later years (1980's), an automated "compact" version of the NPL primary standard attenuator was commercially manufactured and sold by Techtest, Ltd., although the precision guides were still fabricated and measured at NPL. Weinschel Engineering developed and marketed a similarly compact automated dual-frequency WBCO attenuator during this period. Taken in their entirety, this group still remains as world standards to which most automated attenuation measurements are ultimately traced.

\section{THEORY}

A cutoff attenuator may be designed for either a TM or a TE mode of transmission. In designs using the TM mode the guides are usually terminated in some form of disk where the coupling is capacitive. In designs using the TE mode the guides are terminated in a coil or loop and the coupling is inductive.

A waveguide section, excited in one mode by a sinusoidal signal at a frequency below cutoff, has an exponential decay of field strength along its axis. The rate of decay may be closely 
predicted from a knowledge of the cross-sectional dimensions of the guide. A moving probe which couples to the field will therefore have a predictable output variation. The entire assembly constitutes a standard of attenuation.

Practical WBCO attenuators are usually made from metal tubes of uniform circular cross section for ease of precise fabrication and because excitation symmetry is not critical. Circular WBCO attenuators are operated in the dominant $\mathrm{TE}_{11}$ mode. It is true that the dominant $\mathrm{TE}_{11}$ mode in the circular guide is not far separated in frequency from higher undesired modes, but these can be suppressed by metallic strips or dielectric mode filters. In this type of attenuator the $\mathrm{TM}_{01}$ mode is most likely to cause difficulty since its attenuation rate is close to that of the $\mathrm{TE}_{11}$ mode. Mode filters have been constructed that will suppress the TM modes by over $60 \mathrm{~dB}$ with a corresponding decrease in the $\mathrm{TE}_{11}$ mode less than 0.5 dB [5].

Rectangular cross sections have been used and have the advantages that there is greater separation between the cutoff frequencies of higher modes and that the attenuation rates are relatively greater for the unwanted modes above the dominant $\mathrm{TE}_{10}$ mode. The difficulty and cost of fabricating WBCO attenuators with rectangular cross sections and precise uniformity of dimension have generally precluded their use. Such ultraprecise rectangular guides are usually only fabricated for use as impedance standards.

A good approximation for the propagation constant of the $\mathrm{TE}_{11}$ mode is given by $(\gamma$ is in $\mathrm{Np} / \mathrm{m})$ [11]

$$
\begin{aligned}
& \gamma=\frac{S_{11}}{r} \sqrt{1-\left(\frac{2 \pi r f \sqrt{\epsilon}}{S_{11} c}\right)^{2}-\frac{1}{r \sqrt{\pi \mu f \sigma}}} \\
& \left(1+j\left\{1-\frac{1}{1-\frac{1}{r\left[2-\left(\frac{2 \pi r f \sqrt{\epsilon}}{S_{11} c}\right)^{2}\right] \sqrt{\pi \mu f \sigma}}}\right\}\right)
\end{aligned}
$$

where

$S_{11} \approx 1.8411838$ first root of the first derivative of the first-order Bessel function of the first kind;

$r$ radius of waveguide cylinder;

$f \quad$ frequency in Hertz;

$\epsilon$ dielectric constant of the medium inside the waveguide (relative, usually $\approx 1.0003$ for air);

c $\quad \approx 2.997925 \times 10^{8} \mathrm{~ms}$ velocity of light;

$\mu$ permeability of guide wall (relative, usually taken to be 1.00000$)$;

$\sigma \quad$ conductivity of guide wall.

An examination of the equation shows that the most important considerations other than radius are frequency and conductivity. Brown [7] shows that the quantity in square brackets contributes negligible phase shift at IF frequencies in waveguides constructed of good conducting materials such as brass and copper. The actual phase shift is thus typically less than $1^{\circ}$ in $100 \mathrm{~dB}$ of attenuation.

At lower frequencies, skin effect increases the effective electrical radius of the guide and contributes large uncertainties, especially since machining affects wall conductivity. For this reason and the possibility of leakage through the attenuator walls, WBCO attenuators are seldom used below $1 \mathrm{MHz}$. The upper frequency limit is determined by the cutoff frequency and the necessity to operate well below cutoff to decrease the frequency dependence of the attenuator. At extremely high frequencies (above $1 \mathrm{GHz}$ ), required guide dimensions become so small that the necessary mechanical tolerances for an accurate standard cannot be readily achieved.

Equation (1) requires dominant mode operation, which can be achieved by proper excitation and adequate mode suppression or filtering. The coupling inductors used in the attenuator must be sufficiently separated at all times to prevent mutual coupling and loading effects from excessively affecting the excited mode [11]. A separation equivalent to $30 \mathrm{~dB}$ (insertion loss) will make this effect negligible. This is approximately a linear separation equal to one guide diameter. Methods to reduce this minimum insertion loss through feedback to maintain a constant current in the excitation coil or that use compensating networks to minimize interaction loading have been partially successful [11], [14] but at the cost of added complexity.

The remaining important considerations for the circular WBCO attenuator relate to dimensional tolerances in the guide and the resolution and accuracy with which the relative displacement of the exciting and pickup coils can be measured. The guide must not only be almost perfectly circular but also must remain constant in diameter. If any ellipticity exists in the guide, the propagation constant will be different from the circular case, degenerate modes may be excited, and, further, if the major axis has angular variations, this will produce deviations from the exponential law. Typical commercially available WBCO attenuators for use at IF frequencies have inside guide diameters ranging from $0.75-1.5$ in $(1.905-3.810 \mathrm{~cm})$ for corresponding attenuation rates of 40-20 dB/in (1.813-0.907 $\mathrm{Np} / \mathrm{cm}$ ) displacement of the moving coil. Keeping uncertainties to $0.005 \mathrm{~dB}$ per $10-\mathrm{dB}$ increment requires tolerances of a few ten-thousandths of an inch. Tolerances of the same order are required in measuring displacement. The traditional lead screw and mechanical counter have been replaced by precision ruled scales and optical encoding devices. Much greater precision and repeatability has been achieved using interferometry and digital encoding and processing of ambient parameters. Fig. 1 depicts a graph based on (1) with a perfectly conducting guide operated well below cutoff. It shows the relationships of size and attenuation rate for a typical WBCO attenuator.

It is extremely satisfying to note that the theoretical computations and corrections for conductivity, permeability, and dielectric constant have been independently verified by several methods, one of the most accurate being a SQUID since it is based on magnetic flux quanta and stands alone as an attenuation measuring device, although it is not the most convenient to use [15], [17]. 


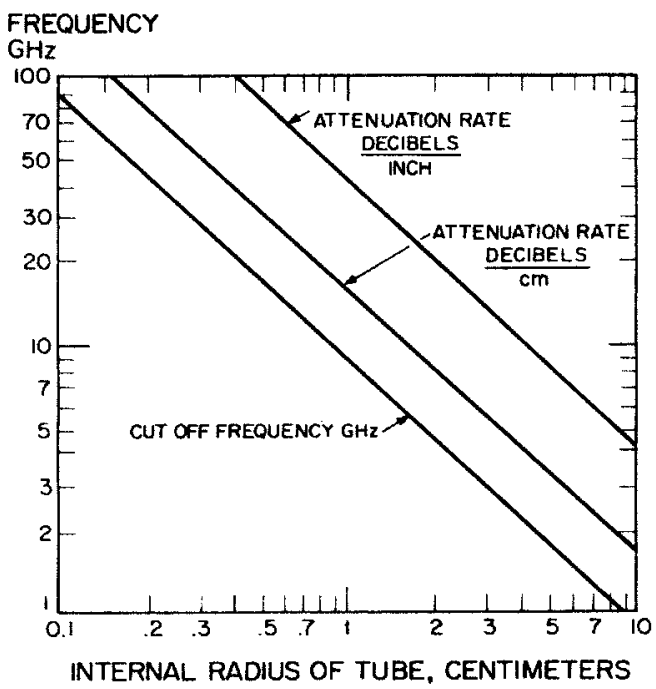

Fig. 1. WBCO size versus attenuation rate.

\section{THE DEvices}

Most WBCO attenuators of extreme precision and accuracy have been constructed to operate at or near $30 \mathrm{MHz}$, a commonly used IF frequency that also requires less skin depth correction than lower frequencies and is sufficiently far enough below cutoff frequencies for practical guide diameters that minimal frequency correction is needed. In recent years 1.25 $\mathrm{MHz}$ has been used as a reference frequency for some automated vector network analyzers and WBCO attenuators have been built to accommodate this frequency and have been calibrated using a repeatable step of attenuation [26], [27]. This lower frequency requires larger corrections for skin depth but has been most successfully used in the Weinschel dual (1.25/30 $\mathrm{MHz}$ ) frequency WBCO attenuator [25].

In the following paragraphs, descriptions are given of WBCO attenuators which were fabricated in the United States, England, and Australia and represent the vast majority of precision devices of primary standards quality.

In the United States, NIST (NBS) in the U.S. Department of Commerce and Weinschel Engineering in the private sector both devised and fabricated true state-of-the-art WBCO devices and systems for their optimal utilization.

\section{A. United States-NIST (NBS)}

Historically, the WBCO attenuators fabricated at NIST progressed from devices with guide diameters of .5 in and a corresponding attenuation rate of about $60 \mathrm{~dB} /$ in through a series of devices with guide diameters of about 1.5 in with an attenuation rate of $20 \mathrm{~dB} /$ in to the current national primary reference standard with a guide diameter of 3.2 in and an attenuation rate of $10 \mathrm{~dB} / \mathrm{in}$.

The earliest ( $60 \mathrm{~dB} /$ in) models were totally dependent upon a finely machined lead screw, a mechanical turns counter, and a circular ruled dial for reading out incremental attenuation. These could be read to $0.05 \mathrm{~dB}$ and had accuracies of the same order. It soon became apparent that these devices took too many turns of the adjustment handle and had insufficient resolution for standards work.
The next series of devices moved to $20 \mathrm{~dB} /$ in of displacement and were refined (several models were copied and sold by private industry) over three decades from the mid-1950's to the mid-1980's. To solve the adjustment speed problems, the precision lead screw was combined with a rack and detent mechanism to permit steps of $10 \mathrm{~dB}$ and fine adjustment from each detent point. The lead screws became ever more precise and the readout system employed preloaded ball-bearing nuts, ultraprecise gear trains, and motor drives for rapid setting.

Even these devices were not quite adequate for secondary standards lab references, and additional improvements were made by incorporating precision ruled scales and optical readouts to permit direct reading to $0.001 \mathrm{~dB}$ and interpolation to $0.0001 \mathrm{~dB}$. Accuracies approached $0.005 \mathrm{~dB}$ per 10 $\mathrm{dB}$ increment. Some of the final versions of these WBCO attenuators were even retrofitted with corner cube reflectors and linear position encoding interferometers to squeeze the ultimate precision from these devices.

As these models were continually improved, a primary reference $30-\mathrm{MHz} \mathrm{WBCO}$ attenuator was required at NIST to calibrate them. Such an attenuator was designed and fabricated by Allred and Cook in 1960 [11] and updated and reevaluated over succeeding years by Russell, Adair, Marler, and Jargon [18], [19]. The attenuator is vertically mounted, has an attenuation rate of $10 \mathrm{~dB} / \mathrm{in}$ with a guide diameter of $3.19725 \mathrm{in}$, and utilizes a laser interferometer for displacement measurement.

The system utilizing this attenuator for measurement of other standards is unique throughout the world in that it is an unmodulated two-channel system that places the test attenuator in series with the standard, thus maintaining constant null sensitivity at the detector. It incorporates a precision trombonetype phase shifter to obtain null balance in phase while the attenuator obtains null balance in amplitude [11]. The null detector is an extremely sensitive receiver employing quadrature detection, thus providing information for digital control of the nulling process [20].

\section{B. United States-Weinschel Engineering}

A series of precision WBCO attenuators and associated attenuation measurement systems were manufactured and marketed at the same time that NIST was developing its series of devices. Although the Weinschel designs employed circular guide, they were fabricated of stainless steel rather than the brass and copper of NIST, NPL, and NML. This required a larger correction for the conductivity term in (1) as well as a permeability correction. The material had two substantial advantages over other materials - it could be honed with superior precision and it was very stable over time. The uncertainties associated with these attenuators were comparable to the secondary standards developed at NIST and were of the order of $0.001 \mathrm{~dB}$ per $10 \mathrm{~dB}$. The measurement system normally used with these WBCO standards is also dual-channel, but puts the test attenuator in the parallel channel, which is then balanced in amplitude with the standard. Amplitude balance is measured by square-wave modulation of the RF signal, which is then detected as an amplitude null at the modulating frequency. This scheme avoids the range-limiting effects of 
putting the test attenuator in series with the standard, but creates additional complexity due to the modulation requirement and the constantly changing sensitivity of the null detector [14]. As usual in technology, tradeoffs are always required.

In the 1980's Weinschel developed a much more accurate $(0.0002-0.0003 \mathrm{~dB}$ per $10 \mathrm{~dB})$ dual-frequency $(1.25 / 30 \mathrm{MHz})$ attenuator that was computer-controlled, employed a laser interferometer for measurement, and featured built-in correction for ambient variations in temperature and barometric pressure [25], [26].

\section{Australia-NML}

Two of the most significant WBCO attenuators of primary standards laboratory quality were developed at the NML in Australia. The first, by Hollway and Kelly [13], was developed in 1963, featured a silver-layered guide with an attenuation constant of $20 \mathrm{~dB} / \mathrm{in}$, and operated at a frequency of 31.25 $\mathrm{MHz}$. Displacement was measured with a precision ruled scale combined with an optical readout. Uncertainty in measuring a 10-dB step was estimated to be $0.001 \mathrm{~dB}$.

The second attenuator, using an electroformed guide and a laser interferometer for displacement measurement, was described in 1984 and yielded a resolution of $0.0001 \mathrm{~dB}$ with an estimated uncertainty of $0.0005 \mathrm{~dB}$ in $10 \mathrm{~dB}$. This attenuator was vertically mounted like the NIST standard and was one of the first described to use remote positioning and control implementing the IEEE 488 computer bus [28].

\section{United Kingdom-NPL}

The NPL in England developed a similar WBCO attenuator using a laser interferometer and an air-supported moving piston to eliminate wear in the circular electroformed copper guide. The moving piston and coil assembly was unique in incorporating an RF oscillator which eliminated the problems associated with flexible coaxial cables. A diode detector and feedback loop were incorporated to ensure a constant RF level to better than $0.0002 \mathrm{~dB}$ over $10 \mathrm{~min}$. A second unique feature associated with the moving "air-bearing" piston was its ability to be used as a precision air gauge for checking the dimensions of the electroformed copper guide [29].

This nonportable design was modified by Holland and Yell into a compact "folded" horizontal design using a laser interferometer and air-bearing piston during the late 1970's, which subsequently formed the basis in the mid-1980's for a commercial version manufactured by Techtest, Ltd., designated as model 310 [30]. This attenuator is remotely controllable over an IEEE 488 interface controlling a high-speed stepping motor which permits adjustment in steps of 0.0001 $\mathrm{dB}$ over a range of $120 \mathrm{~dB}$. This guide has a diameter of about 2 in corresponding to an attenuation constant of 16 $\mathrm{dB} / \mathrm{in}$. The estimated uncertainty is approximately $0.0002 \mathrm{~dB}$ in $10 \mathrm{~dB}$.

\section{E. Validation of Uncertainty}

One of the key issues in determining the accuracy of WBCO attenuators is how to cross-check the uncertainty of the value assigned to the attenuation constant computed using basic EM

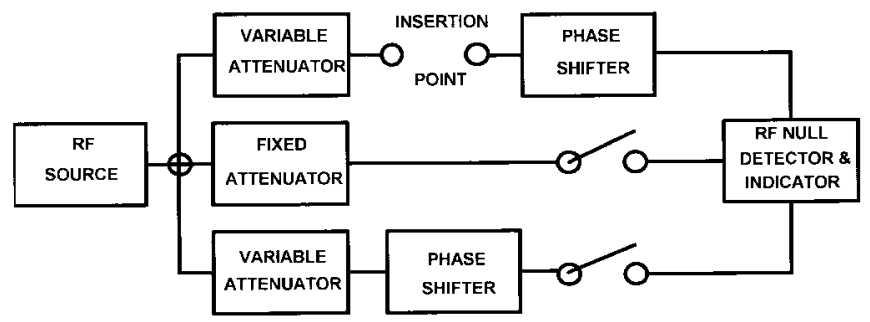

Fig. 2. Laverick self-calibrating attenuation measurement system.

theory and estimated or measured guide size, conductivity, and permeability. To carry this out, various schemes have been used over the years for independent assessment of attenuators.

In the measurement of attenuation, a standard is not actually required as it is basically the determination of a dimensionless ratio. Methods devised for determining attenuation without a standard can be tedious; however, they are valuable when an adequate standard is not available. Most of these methods are based on obtaining known signal ratios by "power division," adding, and subtracting of signals. About the same time, two similar methods were independently developed by Allred [31] and Laverick [32]. These two methods require three branch systems and are shown in Figs. 2 and 3. In the Laverick system, the signals in the three branches are adjusted for equal output by nulling the upper arm where the "unknown" is inserted against each of the other two arms using the phase shifters and variable attenuators. The three branches are connected together and the attenuator to be calibrated in the "unknown" branch is set for a new null. This corresponds to a 6.0206-dB change in attenuation. Repeating the operation by switching the fixed branch in and out yields attenuation increments of $3.5218 \mathrm{~dB}, 2.4988 \mathrm{~dB}$, etc. (voltage ratios of $2,3 / 2,4 / 3$ ).

The method of Allred is more complex and involves solving a set of simultaneous equations, but it yields phase as well as magnitude of the propagation constants of the variable (usually WBCO) attenuators. The procedure is to balance the bridge for a null with the insertion point closed and to note the readings $y_{a}$ and $z_{a}$ of the two attenuators. Phase shifter $\phi 2$ is normally adjusted so that the output voltages of the two variable attenuators are approximately $90^{\circ}$ apart. The unknown is inserted and the attenuators adjusted for a new null without disturbing either phase shifter. The two new attenuator settings $y_{b}$ and $z_{b}$ together with $y_{a}$ and $z_{a}$ are inserted in the following equation involving the insertion ratio of the unknown $K$ :

$$
K=\frac{\varepsilon^{-\gamma_{y} Y_{a}}+W \varepsilon^{-\gamma_{z} Z_{a}}}{\varepsilon^{-\gamma_{y} Y_{b}}+W \varepsilon^{-\gamma_{z} Z_{b}}}
$$

where $\gamma_{y}$ and $\gamma_{z}$ are the propagation constants of the two attenuators and $W$ is a constant of the system expressing the relative phase angle and relative magnitude of the output voltages of the two attenuators. By changing phase shifter $\phi 1$ to a new setting and repeating the procedure, a set of simultaneous equations is obtained involving the same insertion ratio $K$. These equations may then be used to solve for the unknowns $\gamma_{y}, \gamma_{z}, K$, and $W$. 


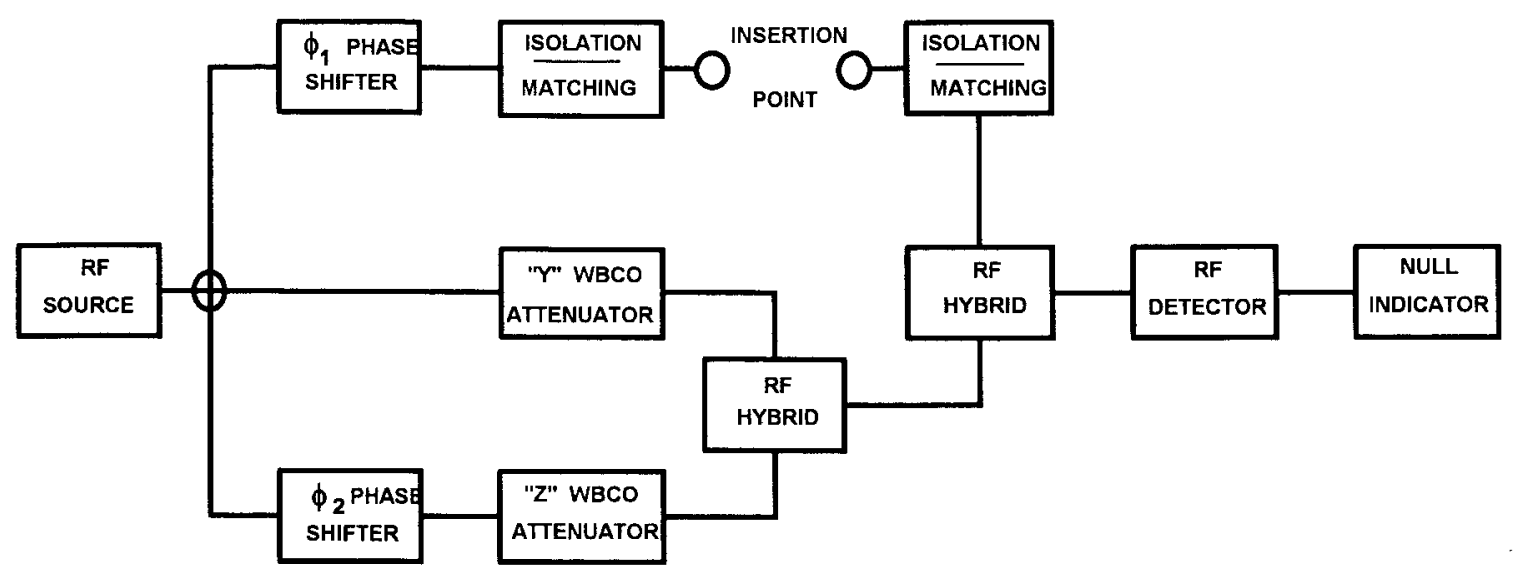

Fig. 3. Allred three-channel self-calibrating attenuation measurement system.

Other "absolute" methods have been described by Davies in 1962 and by Somlo in 1978 [33], [34]. A number of sources of error are present with all of these systems of measurement even though those associated with the standard of attenuation have been eliminated. For example, there are reflections at the insertion point of the test attenuator that cause errors and changes in reflection that affect the power division between channels. Further, there are minor changes due to phase shift adjustment, and limited resolution can cause cumulative small errors to creep in with each step of the measurement process.

Some of the other approaches involve direct power ratio measurement using dc substitution and a truly innovative approach using fixed voltage steps based upon the Josephson junction effect (SQUID's) [17].

In direct power ratio or de substitution some sort of converter is ordinarily used to produce a change in dc power as a function of the RF power to be measured. It is necessary to know precisely how much the converter deviates from its ideal "law" in order to obtain accurate results. A dc substitution technique was used by Engen and Beatty employing bolometers (thermistors) in a balanced bridge arrangement to measure low values of attenuation with extreme precision $(0.0001 \mathrm{~dB})$ [23]. Other variations on this technique employed by Stelzreid yielded similar precision in evaluating standards of attenuation [24].

A totally independent method of evaluating the uncertainty of a precision WBCO attenuator using a SQUID was employed at NIST in 1974 by Kamper, Hoer, and Adair [17]. A SQUID is a loop of superconducting metal closed by a weak point contact called a Josephson junction. Operating in liquid helium, it converts variations in magnetic flux to periodic variations in impedance which are sensed at microwave frequencies. Changes in input RF are indicated as a series of nulls at a microwave frequency which are counted and their values computed from a table of Bessel functions. Using a carefully calibrated NIST WBCO attenuator as the test device, the values for its attenuation increments computed from the number of nulls agreed to better than $0.002 \mathrm{~dB}$ over a $50-\mathrm{dB}$ range. The agreement between the values assigned to the WBCO attenuator and the values measured in the SQUID system were so close that it was difficult to say which system was testing which (Fig. 4).

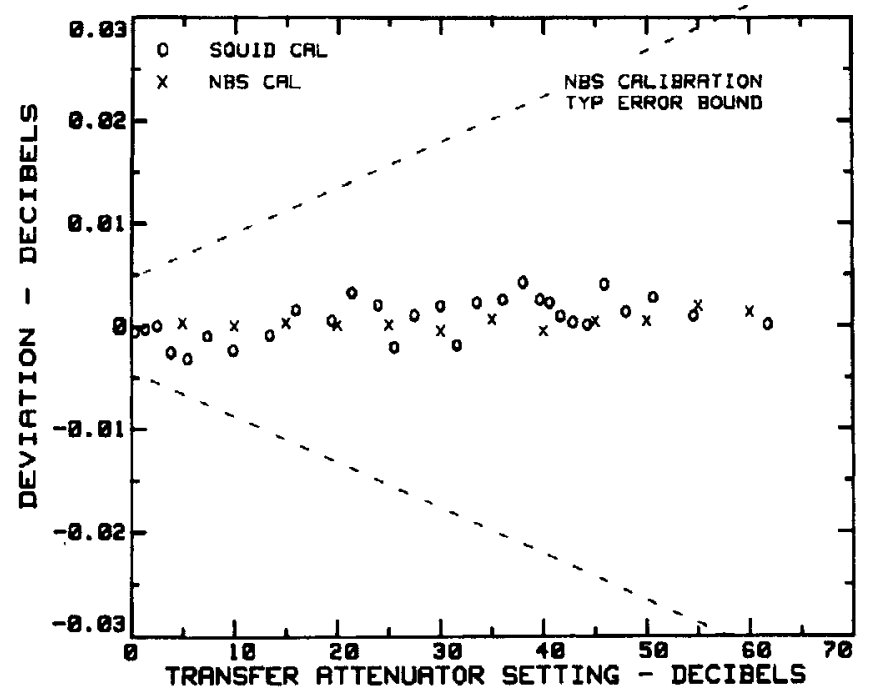

Fig. 4. WBCO comparison with SQUID.

A major attenuation intercomparison at $30 \mathrm{MHz}$ was conducted in 1978 under the auspices of the Bureau of International Weights and Measures (BIPM) and thoroughly validated the theory underlying the uncertainties assigned to these devices in primary laboratories all over the world [35]. Other than a few offsets due, in retrospect, to experimental error, the results of the intercomparison show amazing agreement among the participating laboratories which included France, Germany, Hungary, Sweden, England, Australia, the U.S., and Japan.

\section{CONCLUSIONS}

The WBCO attenuator has established itself as a primary standard of great dynamic range, which was brought to a peak in design technology in the 1980's.

Limitations on the physical properties of materials, escalating costs, and the advent of vector network analyzers have essentially terminated further refinements in the design and implementation of these devices. At this time, their use in ultimate form is confined to the primary standards laboratories.

A review of their development history is a review of the history of standardization and measurement itself. 


\section{REFERENCES}

[1] D. E. Harnett and N. P. Case, "The design and testing of multi-range receivers," Proc. IRE, vol. 23, pp. 578-593, June 1935.

[2] R. N. Griesheimer, "Microwave attenuators. Cutoff attenuators," in Technique of Microwave Measurements (M.I.T. Radiation Laboratory Series). New York: McGraw-Hill, 1947, vol. 11, pp. 685-719.

[3] G. F. Gainsborough, "A method of calibrating standard-signal generators and radio frequency attenuators," Proc. Inst. Elect. Eng., vol. 94, pt. C, pp. 203-210, May 1947.

[4] J. J. Freeman, "Theory and design of a cavity attenuator," J. Res. Nat. Bur. Stand., vol. 40, pp. 235-243, Mar. 1948.

[5] R. E. Grantham and J. J. Freeman, "A standard of attenuation for microwave measurements," Trans. AIEE, vol. 67, pt. I, pp. 329-335, June 1948.

[6] H. A. Wheeler, "The piston attenuator in a waveguide below cutoff," Wheeler Labs., Great Neck, NY, Mono. 8, Jan. 1949.

[7] J. Brown, "Corrections to the attenuation constants of piston attenuators," Proc. Inst. Elect. Eng., vol. 96, pt. C, pp. 491-495, Nov. 1949.

[8] H. M. Barlow and A. L. Cullen, Microwave Measurements. London: Constable, 1950, pp. 242-254, 384-388.

[9] C. M. Allred, "Chart for the $\mathrm{TE}_{11}$ mode piston attenuator," J. Res. Natl. Bur. Stand., vol. 48, pp. 109-110, Feb. 1952.

[10] D. M. Kerns and R. W. Hedburg, "Propagation constant in rectangular waveguide of finite conductivity," J. Appl. Phys., vol. 25, pp. 1550-1551, Dec. 1954.

[11] C. M. Allred and C. C. Cook, "A precision RF attenuation calibration system," IRE Trans. Instrum., vol. I-9, pp. 268-274, Sept. 1960.

[12] L. A. Birger, "Nonlinearity of cutoff attenuators due to the effect of loading," Ismer. Tekh., no. 11, pp. 59-61, 1960.

[13] D. L. Hollway and F. P. Kelly, "A standard attenuator and the precise measurement of attenuation," IEEE Trans. Instrum. Meas., vol. IM-13, pp. 33-44, Mar. 1964

[14] B. O. Weinschel and G. U. Sorger, "Waveguide below-cutoff attenuation standard," Acta IMEKO III, pp. 407-424, 1964

[15] R. A. Kamper, M. B. Simmonds, R. T. Adair, and C. A. Hoer, "Quantum mechanical measurement of RF attenuation," in Proc. 1972 Applied Superconductivity Conf., pp. 696-700.

[16] R. A. Kamper, M. B. Simmonds, C. A. Hoer, and R. T. Adair, "Measurement of RF power and attenuation using superconducting quantum interference devices," Natl. Bur. Stand., Boulder, CO, Tech. Note 643, pp. 12-63, Aug. 1973.

[17] R. T. Adair, M. B. Simmonds, R. A. Kamper, and C. A. Hoer, "RF attenuation measurements using quantum interference in superconductors," IEEE Trans. Instrum. Meas., vol. IM-23, no. 4, pp. 375-381, Dec. 1974

[18] R. T. Adair and D. H. Russell, "A calibration service for $30 \mathrm{MHz}$ attenuation and phase shift," Nat. Bur. Stand. Measurement Services Special Publication, no. 250-32, pp. 3-1, 3-26, Apr. 1988.

[19] J. A. Jargon, "A revised uncertainty analysis for the NIST 30-MHz attenuation calibration system," in Measurement Science Conf., Pasadena, CA, 1994.

[20] R. E. Stoltenberg, "RF null detector NBS/SND," Nat. Bur. Stand., Boulder, CO, Internal Rep. 73-302, June 1973.

[21] J. A. Jargon, "A $30 \mathrm{MHz}$ comparison receiver," in Asia-Pacific Microwave Conf. Proc., 1995, vol. 1, pp. 94-96.
[22] D. H. Russell and W. Larson, "RF attenuation," Proc. IEEE, vol. 55, no. 6, pp. 942-959, June 1967

[23] G. F. Engen and R. W. Beatty, "Microwave attenuation measurements with accuracies from 0.0001 to 0.06 decibel over a range of 0.01 to 50 decibels," J. Res. Nat. Bur. Stand., vol. 64C, pp. 139-145, Apr.-June 1960.

[24] C. T. Stelzried, M. S. Reid, and S. M. Petty, "A precision dc potentiometer microwave insertion loss test set," IEEE Trans. Instrum. Meas., vol. IM-15, pp. 98-104, Sept. 1966.

[25] B. O. Weinschel, "A new attenuation standard uses laser interferometer," Microwave J., vol. 27, no. 8, pp. 145-148, Aug. 1984.

[26] R. C. Powell, "Calibration of a waveguide below cutoff attenuation standard using a repeatable attenuation step," in Conf. Precision Electromagnetic Measurements, June 1986, pp. 116-118.

[27] R. A. Ginley and C. M. Allred, "1.25-MHz attenuation measurement system," IEEE Trans. Instrum. Meas., vol. IM-35, no. 4, pt. I, pp. 463-466, Dec. 1986.

[28] T. E. Cousins and H. Kobler, "A 30-MHz standard attenuator," JEEE Australia-IE Aust. \& IREE Aust., vol. 4, no. 1, pp. 1-5, Mar. 1984.

[29] J. M. Steele, C. R. Ditchfield, and A. E. Bailey, "Electrical standards of measurement, Part 2: RF and microwave standards," Proc. Inst. Elect. Eng.. IEEE Reviews, vol. 122, no. 10R, pp. 1037-1053, Oct. 1975.

[30] K. P. Holland and R. W. Yell, "A precision automated measurement system based on a waveguide below-cutoff attenuator," British Electromagnetic Proc., 1978.

[31] C. M. Allred, "Self-calibrating method of measuring insertion ratio," Nat. Bur. Stand. Tech. News Bull., vol. 41, pp. 132-133, Sept. 1957.

[32] E. Laverick, "The calibration of microwave attenuators by an absolute method," IRE Trans. Microwave Theory Tech., vol. MTT-5, pp. 250-254, Oct. 1957.

[33] M. C. Davies, "An absolute method for calibrating microwave attenuators," Proc. Inst. Elect. Eng. vol. 109, pt. B, supp. 23, pp. 704-712, May 1962.

[34] P. I. Somlo, "A voltage doubling circuit for the absolute calibration of $30 \mathrm{MHz}$ attenuation measurement systems," IEEE Trans. Instrum. Meas., vol. IM-27, pp. 76-79, Mar. 1978.

[35] Rapport de la $15^{e}$ Session, Comité Consultatif d'Electricité; Bureau International des Poids et Mesures, Annexe E15, E81-E94, 1978.

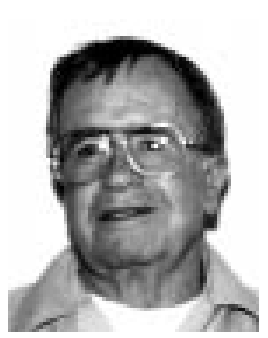

David H. Russell is a microwave/millimeter wave engineer who retired from the Electromagnetic Fields Division, National Institute of Standards and Technology (NIST), Boulder, CO, in 1993. As a Group Leader, he directed the development of power, noise, impedance, and attenuation standards, and their application in automated measurement systems. His group also did pioneering work in design and fabrication of on-chip standards for monolithic microwave integrated circuits (MMIC) device metrology. During 38 years at NIST, his research interests were primarily in standards and systems for microwave and millimeter wave attenuation metrology in coaxial, uniconductor, and planar waveguides. 